\title{
A comparative analysis of the observed effects of 2D tunneling bifurcations for quasi-one-dimensional and quasi-two-dimensional Au-QD systems in an external electric field
}

\author{
M. B. Semenov ${ }^{1}$, V. D. Krevchik ${ }^{1}$, O. N. Gorshkov², D. O. Filatov², Y. Dakhnovsky ${ }^{3}$, A. V. Nikolaev ${ }^{4}$, \\ A. P. Shkurinov ${ }^{4}$, V. Yu. Timoshenko ${ }^{4}$, P. V. Krevchik ${ }^{1}$, A. K. Malik ${ }^{5}$, Y. H. Wang ${ }^{6}$, T. R. Li ${ }^{6}$, Y. Zhu $^{7}$, \\ S. Zhuang ${ }^{7}$, R. V. Zaytsev ${ }^{1}$, I. S. Antonov ${ }^{1}$, I. M. Semenov ${ }^{1}$, A. K. Aringazin ${ }^{8}$, A. V. Shorokhov ${ }^{9}$ \\ ${ }^{1}$ Department of Physics, Penza State University, Penza 440026, Russia \\ ${ }^{2}$ Department of Physics, Lobachevsky State University of Nizhny Novgorod, \\ Nizhny Novgorod 603950, Russia \\ ${ }^{3}$ Department of Physics and Astronomy, University of Wyoming, WY 82071 Laramie, USA \\ ${ }^{4}$ Moscow State University, Moscow 119991, Russia \\ ${ }^{5}$ Department of Physics, Multanimal Modi College Modinagar, Uttar Prasesh 201204, India \\ ${ }^{6}$ Institute of Functional and Environmental Materials, Lanzhou University, Lanzhou, China \\ ${ }^{7}$ University of Shanghai for Science and Technology, Shanghai 200093, China \\ 8 Institute for Basic Research, Eurasian National University, Astana 010008, Kazakhstan \\ ${ }^{9}$ Institute of Physics and Chemistry, National Research Mordovia State University, Saransk 430005, Russia \\ misha29.02@gmail.com, alex.shorokhov@mail.ru
}

PACS 73.40.Gk, 03.65.Xp

DOI 10.17586/2220-8054-2018-9-6-724-734

\begin{abstract}
The effects of 2D tunneling bifurcations for quasi-one-dimensional and quasi-two-dimensional Au-quantum dot (QD) arrays in thin dielectric films in an external electric field have been studied theoretically and experimentally by Conductive Atomic Force Microscopy (CAFM). In the case of quasi-one-dimensional $\mathrm{Au}-\mathrm{QD}$ structures (with the QD size $\sim 5 \mathrm{~nm}$ ), in a dielectric film, a single break under positive bias polarity, corresponding to the effect of $2 \mathrm{D}$ tunneling bifurcation, previously predicted theoretically by our team [1], has been detected in experimental I-V curves of the CAFM probe-to-sample contact. A convincing qualitative agreement between the obtained experimental I-V curves and the theoretical field dependence for the 2D-dissipative tunneling probability in the model 2D-oscillator potential has been obtained for the case of parallel tunneling in the weak-dissipation limit at a finite temperature in an external electric field. In the case of quasi-two-dimensional structures with Au QD (with the QD sizes of 2 to $5 \mathrm{~nm}$ ), possessing metamaterial properties, a pair of kinks corresponding to the double effect of $2 \mathrm{D}$-tunneling bifurcations has been detected on the experimental $\mathrm{I}-\mathrm{V}$ curves. A qualitative agreement between the experimental I-V curves and the theoretical field dependence for the 2D-dissipative tunneling probability has been obtained for a situation with an effectively "negative" permittivity of the heat bath.
\end{abstract}

Keywords: quantum tunneling with dissipation, quantum dots, two-dimensional bifurcations.

Received: 27 October 2018

\section{Introduction}

The first mention of the 2D-tunneling bifurcations effect was in the work of Yu. N. Ovchinnikov and B. I. Ivlev [2] under consideration of two-dimensional systems with interacting Josephson junctions. The authors assumed that this effect can be observed in the form of a characteristic break in the corresponding tunnel I-V curves, but its observation proved difficult due to the washout of this feature by current noise in the vicinity of the bifurcation point. The unstable effect of 2D-bifurcations for the case of antiparallel tunneling transport turned out to be observable in the form of a kink on the temperature dependence of the chemical reaction rate of the interacting protons transfer in a porphyrin- type system. A theory of the 2D-tunneling bifurcations effect in $2 \mathrm{D}$ potentials with different topologies was developed in the works by V. A. Bendersky and co-authors [3].

The effect of 2D-tunneling bifurcations for the parallel transfer of interacting particles in the weak-dissipation limit with allowance for the quantum beat regime in the vicinity of the bifurcation point, predicted by us in an earlier work [1], has been observed experimentally in a quasi-one-dimensional Au-QD array in an external electric field by Conductive Atomic Force Microscopy (CAFM). A description of the relevant experiment is presented in Section 2 of this article [4].

Section 3 is devoted to the recently performed author's experiment on measuring the tunnel I-V curves of the quasi-two-dimensional $\mathrm{Au}-\mathrm{QD}$ arrays, which could potentially manifest the metamaterial properties (particularly, 
a negative refractive index) at certain values of spacing between the Au-QDs in the periodic 2D-arrays [5-7]. In Sections 4 and 5 we give a theoretical calculation of the 2D-dissipative tunneling probability with allowance for 2D-bifurcation modes in the model 2D-oscillator potential at a finite temperature in an external electric field in the weak dissipation limit for both the dielectric matrix case (Section 4) and the case of 2D-structures (dielectric matrix $+\mathrm{Au}-\mathrm{QDs}$ ), which have the properties of a metamaterial (Section 5). In general, the dielectric matrix itself can possess the properties of a metamaterial, but for this it must have a periodic modulation of the refractive index in space, for example, a multilayer structure. However, in this case we often speak of quantum dots in microresonators. Section 6 gives the qualitative comparison of the obtained experimental and theoretical results and compares the effects of 2D tunnel bifurcations both for quasi-one-dimensional and quasi-two-dimensional structures with $\mathrm{Au}-\mathrm{QDS}$.

A qualitative conclusion is drawn that it is possible to experimentally confirm the theoretically predicted effect of 2D-tunneling bifurcations for the investigated nanostructures.

\section{Experimental $\mathbf{I}-\mathbf{V}$ characteristics for quasi-one-dimensional $\mathrm{Au}-\mathrm{QD}$ structures}

In the work by Yu. N. Ovchinnikov [8], it has been shown that the conductivity of granular metallic films is related to the tunneling processes between neighboring granules and that the interaction with the heat bath, ensuring a real transition to states localized in the "neighboring" cluster, is rather small. Thus, the characteristics of the tunnel current in the systems under study can be considered in the limit of comparatively "weak" dissipation, but sufficient to ensure the "decay" of the double-well oscillator potential used in the proposed model. In addition, an appreciable contribution to the tunneling current can be provided by the tunneling probability, estimated to within a pre-exponential factor in Ref. [9].

Figure 1 presents the experimental scheme of the studies and a typical current-voltage (I-V) curves obtained by the experimental group (O. N. Gorshkov, D. O. Filatov, D. A. Antonov) in Research and Educational Center for Physics of Solid State Nanstructures at Lobachevskii State University of Nizhni Novgorod (Russia) [4]. When measuring the tunnel current from the CAFM probe tip to the nearest Au nanocluster (QD), it is quite possible that, due to some inhomogeneities (protrusions) on the tip apex, parallel closely spaced channels of the tunnel current can take place (so called nulti-tip effect). If the sizes of the protrusions are less than the nanocluster (QD) ones, then at a negative bias voltage the asymmetry of the potential along the transfer coordinate changes, and the system becomes a two-dimensional one essentially. Depending on the magnitude of the external electric field strength, as well as on temperature and on relative permittivity of the heat bath, in the parallel synchronous transfer of Coulomb-interacting particles, either stable synchronous or asynchronous transfer takes place. The switching from one mode to another corresponds to a bifurcation point, which will be theoretically described briefly in Section 4 .

One of the characteristic features of the I-V curves, shown in Fig. 1b, is a sharp break observed at positive bias voltages, which is assumed to be due to a change in the tunneling regime along parallel channels in an asymmetric 2D potential or by the presence of a bifurcation point described in [1]. Near this point, a small transition region with a separate feature was observed on the current-voltage curves, which probably can correspond to the quantum beat regime predicted in [1].

Finally, in the negative voltage region, we observed a characteristic single peak, which, as described in Ref. [9], is related to the singularity of the pre-exponential factor under conditions when the model potential becomes symmetric with the change of the external electric field that affects the magnitude of the potential asymmetry parameter.

This set of the theoretically and experimentally studied effects allows us to conclude that it is possible to experimentally observe stable 2D tunneling bifurcations with dissipation, which is one of the main results of this work.

\section{Experimental I-V characteristics for quasi-two-dimensional Au-QD structures}

In the framework of the experimental scheme described in Section 2, a situation was realized where the tunnel current was not formed through a single Au-QD (quasi-one-dimensional case), but through two neighboring AuQDs (quasi-two-dimensional case, Fig. 2). The following tunneling current-voltage characteristics were obtained (Fig. 3), having two "smoothed" kinks. It is assumed that such quasi-two-dimensional structures may have the properties of a metamaterial.

On the experimental curve of Fig. 3b, in addition to the "smoothed" double kinks, the quantum beat regime was also observed in the neighborhood of the points of 2D bifurcations. 


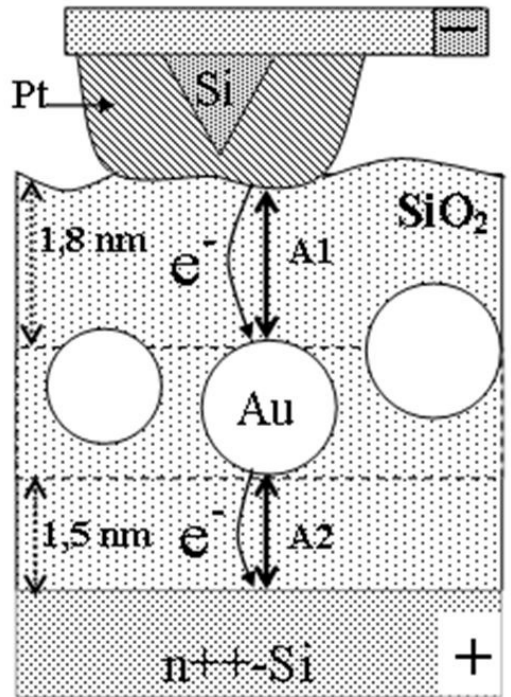

a)

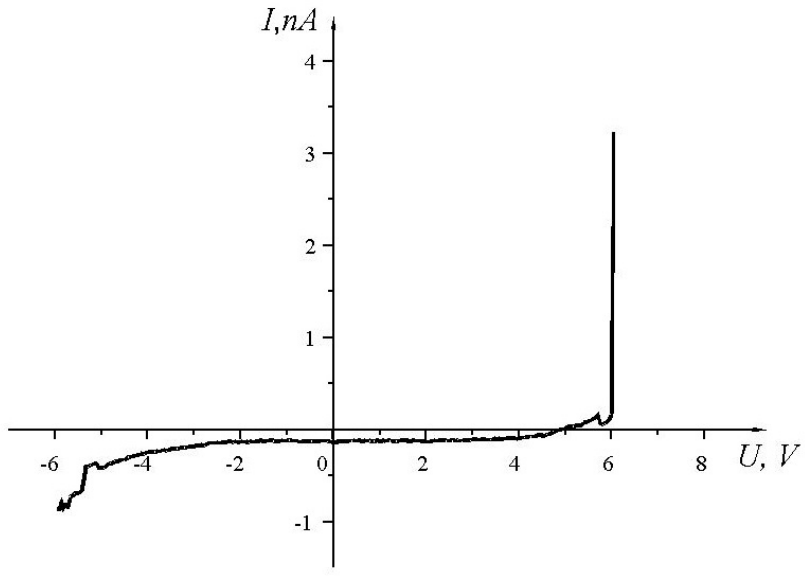

b)

FIG. 1. (a) Schematic representation of the experimental setup for studying the dissipative electron tunneling via an individual $\mathrm{Au}$ nanoclusters embedded into a tunnel-transparent $\mathrm{SiO}_{2}$ film on $n^{+}$-Si substrate by CAFM. $A 1$ is the tunnel-transparent probe-cluster potential barrier, $A 2$ is the cluster-substrate one. (b) An example of I-V curve of a CAFM probe contact to the nanocomposite $\mathrm{SiO}_{2}(1.5 \mathrm{~nm}) / \mathrm{SiO}_{2}: \mathrm{nc}-\mathrm{Au}(1.6 \mathrm{~nm}) / \mathrm{SiO}_{2}(1.8 \mathrm{~nm}) / \mathrm{n}^{+}-\mathrm{Si}(100)$ stack film

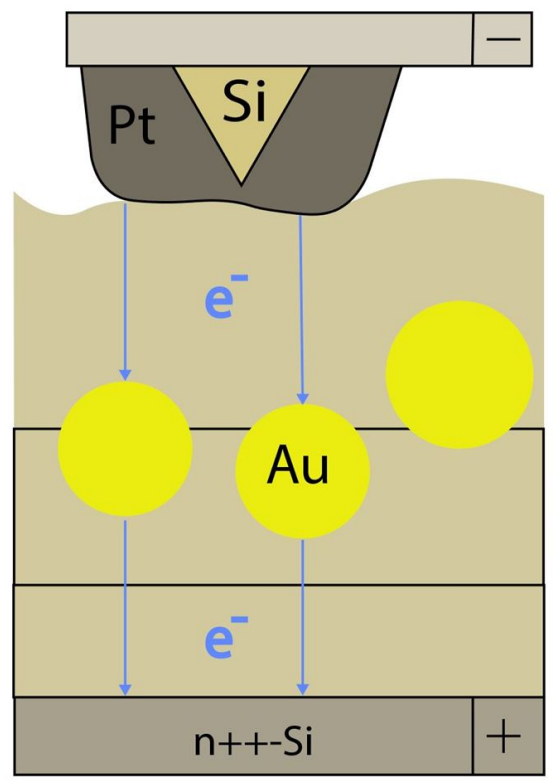

FIG. 2. Schematic representation of the experimental setup for studying the dissipative electron tunneling via double Au-QDs embedded into a tunnel-transparent $\mathrm{SiO}_{2}$ film on $n^{+}$-Si substrate by CAFM. This case corresponds to the quasi-two-dimensional structures, which may have the properties of a metamaterial 


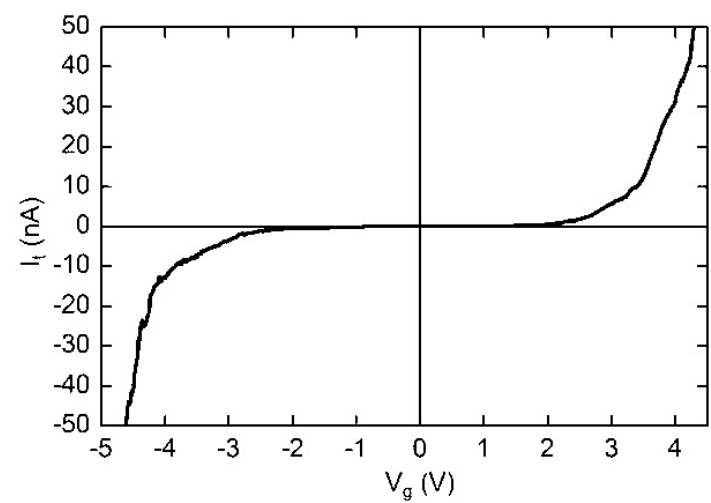

a)

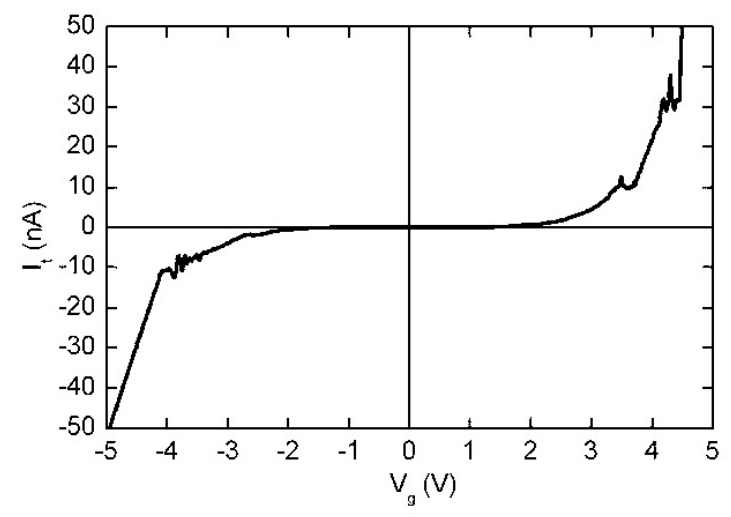

b)

FIG. 3. An example $\mathrm{I}-\mathrm{V}$ curves of a CAFM probe contact to the nanocomposite $\mathrm{SiO}_{2}(1.5 \mathrm{~nm}) / \mathrm{SiO}_{2}$ : double $\mathrm{nc}-\mathrm{Au}(1.6 \mathrm{~nm}) / \mathrm{SiO}_{2}(1.8 \mathrm{~nm}) / n^{+}-\mathrm{Si}$ stack film (Fig. 2): with "smoothed" double kinks + (b, with) and - (a, without) the quantum beat regime

In Section 4, a qualitative comparison of the tunneling current-voltage characteristics given in Fig. 3a and Fig. $3 \mathrm{~b}$ with the field dependence of the 2D-dissipative parallel tunneling probability in case of the quasi-twodimensional structure with properties of a metamaterial, will be presented.

\section{Theoretical calculation of the $2 D$ bifurcation regime in the model $2 D$ oscillator potential at a finite temperature in an external electric field in the weak dissipation limit for the case of a dielectric matrix}

The influence of the electric field $E$ on the asymmetric two-well oscillator potential gives the following expression for $\tilde{U}(q)$

$$
\tilde{U}(q)=\frac{\omega_{0}^{2}}{2}\left(q-b_{0}\right)^{2} \theta(q)+\left[\frac{\omega_{0}^{2}}{2}\left(q+a_{0}\right)^{2}-\Delta I\right] \theta(-q)-|e| E q
$$

where the parameter $\Delta I=\frac{\omega_{0}^{2}}{2}\left(a_{0}^{2}-b_{0}^{2}\right)$ determines the initial asymmetry of the potential in the absence of the field, and, as it is known, leads to a change in the asymmetry parameter proportional to the electric field magnitude.

In order to use the standard model to determine the probability of 2D-dissipative tunneling, we use the following notation for the renormalized two-well oscillator potential in an external electric field: $b=b_{0}+\frac{|e| E}{\omega_{0}^{2}}$, $a=a_{0}-\frac{|e| E}{\omega_{0}^{2}}$.

For the case of parallel tunneling particles, we select the potential energy $U_{1}\left(R_{1}, R_{2}\right)$ in the following form (see $[4,14]$ as also Fig. 4):

$$
\begin{aligned}
U_{1}\left(R_{1}, R_{2}\right)=\frac{\omega^{2}\left(R_{1}+a\right)^{2}}{2} & \theta\left(-R_{1}\right)+\left[-\Delta \mathrm{I}+\frac{\omega^{2}\left(R_{1}-b\right)^{2}}{2}\right] \theta\left(R_{1}\right)+ \\
+ & \frac{\omega^{2}\left(R_{2}+a\right)^{2}}{2} \theta\left(-R_{2}\right)+\left[-\Delta \mathrm{I}+\frac{\omega^{2}\left(R_{2}-b\right)^{2}}{2}\right] \theta\left(R_{2}\right)-\frac{\alpha\left(R_{1}-R_{2}\right)^{2}}{2}
\end{aligned}
$$

where $\Delta I=\frac{1}{2} \omega^{2}\left(b^{2}-a^{2}\right)$ is the "reaction heat" or the asymmetry parameter of the potential; $\alpha(\alpha>0)$ is the coefficient of interaction between particles, which in the dipole-dipole approximation depends on the magnitude of the relative dielectric constant of the heat-bath (or dielectric matrix), $R_{1,2}$ are the parallel coordinates of the tunneling.

Taking into account the interaction of particles tunneling through parallel channels, the potential reconstruction becomes essentially two-dimensional (see Fig. 5). 


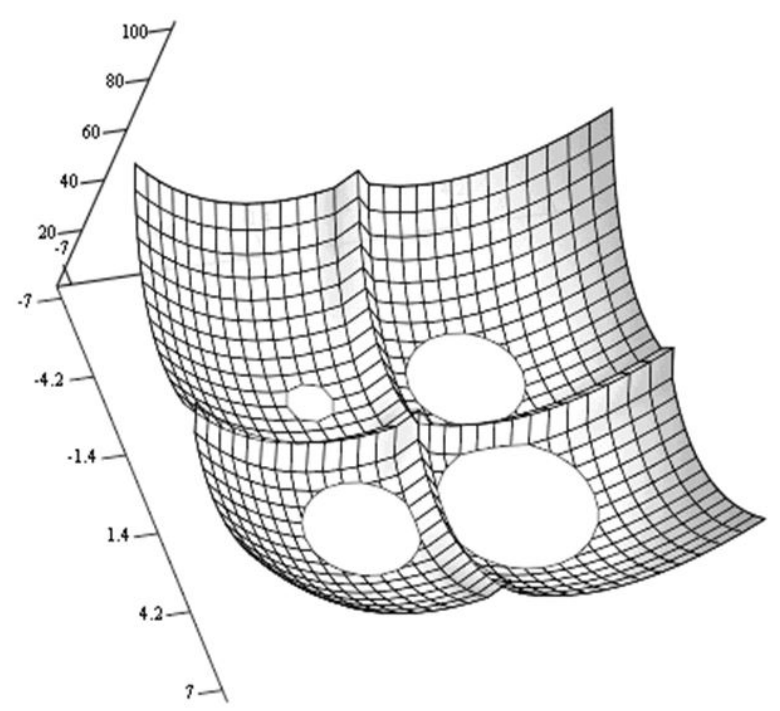

FIG. 4. Surface of the potential energy Eq. (2), (after "switching on" the interaction between particles), as a function of the reaction coordinates for particles moving in parallel coordinates (from catilever needle to the nearest Au-QD), $\left(a=2 ; b=2.5 ; \alpha^{*}=2 \alpha / \omega^{2}=0.0001\right.$ )

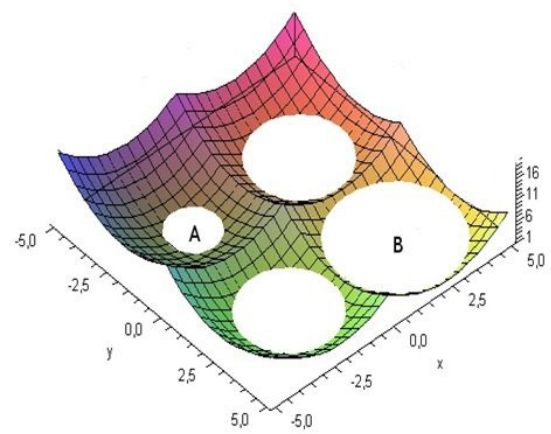

a)

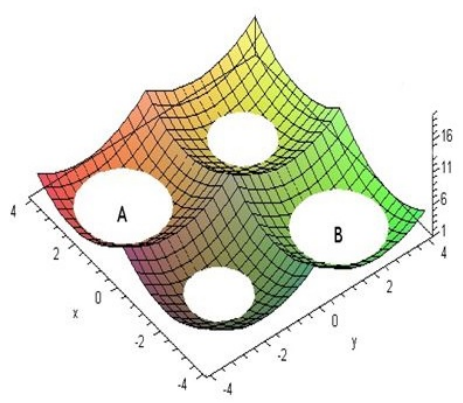

b)

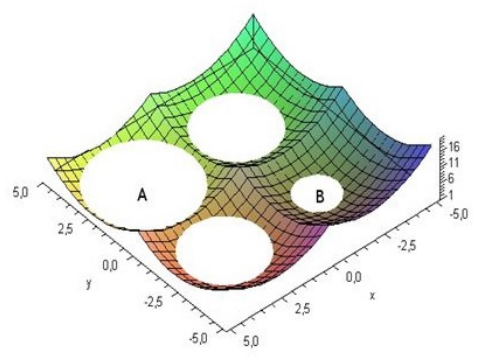

c)

FIG. 5. Change in the asymmetry of the potential energy surface for parallel transport of particles in an external electric field (with negative applied voltage). At a certain value of the applied voltage, the potential becomes symmetrical (b)

In the framework of the standard 2D-model of dissipative tunneling in the weak dissipation limit [1], we obtain

$$
\begin{aligned}
S\left\{R_{1}, R_{2}\right\}= & \int_{-\beta / 2}^{\beta / 2} d \tau\left\{\frac{\dot{R}_{1}^{2}}{2}+\frac{\dot{R}_{2}^{2}}{2}+V\left(R_{1}, R_{2}\right)+\right. \\
& \left.\frac{1}{2} \int_{-\beta / 2}^{\beta / 2} d \tau^{\prime} D\left(\tau-\tau^{\prime}\right)\left[R_{1}(\tau)+R_{2}(\tau)\right]\left[R_{1}\left(\tau^{\prime}\right)+R_{2}\left(\tau^{\prime}\right)\right]\right\}
\end{aligned}
$$

where

$$
D(\tau)=\frac{1}{\beta} \sum_{n=-\infty}^{\infty} D\left(\nu_{n}\right) e^{i \nu_{n} \tau},
$$

$\beta=\hbar / k_{B} T$ is the inverse temperature, ( $\hbar, k_{B}$ and the particle masses, as before, are assumed to be equal to 1$)$; $\nu_{n}=2 \pi n / \beta$ is the Matsubara frequency; and, finally, $D(\tau)$ is the Green's function of phonons. 
A two-dimensional quasiclassical trajectory (instanton), that minimizes the action functional, is determined from the equations of motion

$$
\frac{\delta S}{\delta R_{1}}=0, \quad \frac{\delta S}{\delta R_{2}}=0
$$

We seek a solution of the system of these equations in the following form:

$$
R_{1}(\tau)=\frac{1}{\beta} \sum_{n=-\infty}^{\infty} R_{n}{ }^{(1)} e^{i \nu_{n} \tau}, \quad R_{2}(\tau)=\frac{1}{\beta} \sum_{n=-\infty}^{\infty} R_{n}{ }^{(2)} e^{i \nu_{n} \tau} .
$$

It should be mentioned that the solutions of the equations system (5) are under the assumption of simultaneous start of tunneling particles. The times $\tau_{1}$ and $\tau_{2}$ correspond to the moments of passage for the tops of the barriers by the particles along the corresponding reaction coordinates, and can be found from the following equations:

$$
R_{1}\left(\tau_{1}\right)=0, \quad R_{2}\left(\tau_{2}\right)=0
$$

In the case of parallel motion of tunneling particles (potential energy $U_{1}\left(R_{1}, R_{2}\right)$ (Eq. 2), the action $S$ as a function of the parameters $\tau_{1}$ and $\tau_{2}$ takes the form:

$$
\begin{aligned}
S=2 a(b+a)\left(\tau_{1}+\tau_{2}\right) \omega^{2}- & \frac{\omega^{2}(a+b)^{2}\left(\tau_{1}+\tau_{2}\right)^{2}}{\beta}-\frac{\omega^{4}(a+b)^{2}\left(\tau_{1}-\tau_{2}\right)^{2}}{\left(\omega^{2}-2 \alpha\right) \beta}- \\
& -\frac{2 \omega^{4}(a+b)^{2}}{\beta} \sum_{n=1}^{\infty}\left\{\frac{\left(\sin \nu_{n} \tau_{1}+\sin \nu_{n} \tau_{2}\right)^{2}}{\nu_{n}^{2}\left(\nu_{n}^{2}+\omega^{2}+\xi_{n}\right)}+\frac{\left(\sin \nu_{n} \tau_{1}-\sin \nu_{n} \tau_{2}\right)^{2}}{\nu_{n}^{2}\left(\nu_{n}^{2}+\omega^{2}-2 \alpha\right)}\right\}
\end{aligned}
$$

where $\xi_{n}$ can be assumed to be 0 , if we neglect the interaction with the oscillators of the medium.

We introduce the notations: $\varepsilon=\varepsilon^{*} \omega=\left(\tau_{1}-\tau_{2}\right) \omega ; \quad \tau=2 \tau^{*} \omega=\left(\tau_{1}+\tau_{2}\right) \omega ; \beta^{*}=\beta \omega / 2 ; \quad \alpha^{*}=$ $2 \alpha / \omega^{2} ; \quad b^{*}=b / a, \quad b \geq a$.

If we neglect interaction with phonons (medium oscillators), then $\xi_{n}=0$, and the action $S$ (8) as a function of the parameters $\varepsilon$ and $\tau$ takes the form

$$
\begin{aligned}
S=(a+b) \omega \tau\left(2 a-\frac{a+b}{2}\left[1+\frac{1}{1-\alpha^{*}}\right]\right)+\frac{\omega(a+b)^{2}}{2}(\tau-|\varepsilon|) \frac{\alpha^{*}}{1-\alpha^{*}}- \\
-\frac{\omega(a+b)^{2}}{2}\left\{-\operatorname{coth} \beta^{*}+\frac{1}{\sinh \beta^{*}}\left[\cosh \left(\beta^{*}-\tau\right) \cosh \varepsilon+\cosh \left(\beta^{*}-\tau\right)-\cosh \left(\beta^{*}-|\varepsilon|\right)\right]\right\}- \\
-\frac{\omega(a+b)^{2}}{2\left(1-\alpha^{*}\right)^{3 / 2}}\left\{\operatorname{coth}\left(\beta^{*} \sqrt{1-\alpha^{*}}\right)+\frac{1}{\sinh \left(\beta^{*} \sqrt{1-\alpha^{*}}\right)}\left[\cosh \left(\left(\beta^{*}-\tau\right) \sqrt{1-\alpha^{*}}\right) \times\right.\right. \\
\left.\left.\quad \times \cosh \left(\varepsilon \sqrt{1-\alpha^{*}}\right)-\cosh \left(\left(\beta^{*}-\tau\right) \sqrt{1-\alpha^{*}}\right)+\cosh \left(\left(\beta^{*}-|\varepsilon|\right) \sqrt{1-\alpha^{*}}\right)\right]\right\}
\end{aligned}
$$

We will write expressions $((7))$ in the form

$$
\begin{aligned}
& \sinh \varepsilon\left[\cosh \tau \operatorname{coth} \beta^{*}-\sinh \tau-\operatorname{coth} \beta^{*}\right]+\frac{1}{1-\alpha^{*}} \sinh \left(\varepsilon \sqrt{1-\alpha^{*}}\right) \times \\
& \quad \times\left[\cosh \left(\tau \sqrt{1-\alpha^{*}}\right) \operatorname{coth}\left(\beta^{*} \sqrt{1-\alpha^{*}}\right)-\sinh \left(\tau \sqrt{1-\alpha^{*}}\right)+\operatorname{coth}\left(\beta^{*} \sqrt{1-\alpha^{*}}\right)\right]=0 \\
& 3-\frac{4}{1+b^{*}}-\frac{1}{1-\alpha^{*}}+\cosh \varepsilon\left[\sinh \tau \operatorname{coth} \beta^{*}-\cosh \tau-1\right]+\sinh \tau \operatorname{coth} \beta^{*}- \\
& -\cosh \tau+\frac{1}{1-\alpha^{*}} \cosh \left(\varepsilon \sqrt{1-\alpha^{*}}\right)\left[\sinh \left(\tau \sqrt{1-\alpha^{*}}\right) \operatorname{coth}\left(\beta^{*} \sqrt{1-\alpha^{*}}\right)-\cosh \left(\tau \sqrt{1-\alpha^{*}}\right)+1\right]- \\
& \quad-\frac{1}{1-\alpha^{*}}\left[\sinh \left(\tau \sqrt{1-\alpha^{*}}\right) \operatorname{coth}\left(\beta^{*} \sqrt{1-\alpha^{*}}\right)-\operatorname{coth}\left(\tau \sqrt{1-\alpha^{*}}\right)\right]=0
\end{aligned}
$$

In addition to the always existing solution $\tau_{1}=\tau_{2}$, additional solutions appear in Fig. $6 \mathrm{~b}$ and $6 \mathrm{c}$, (with increasing of $\beta$-parameter or decreasing temperature). Fig. $6 \mathrm{~b}$ shows the moment (bifurcation) of the appearance of an additional solution, more preferable in magnitude of the quasiclassical action. In Fig. 6c, there are already two such additional solutions, but only one of them is preferable in the magnitude of the action (in the case when these solutions are close, the quantum beat regime is realized). 


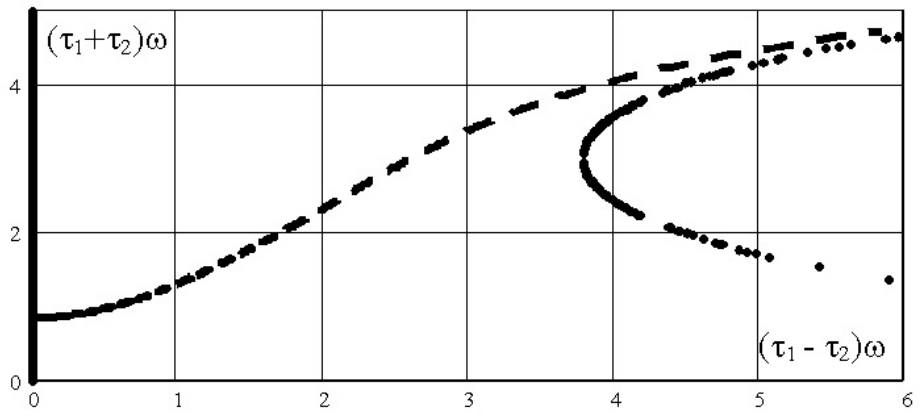

a)

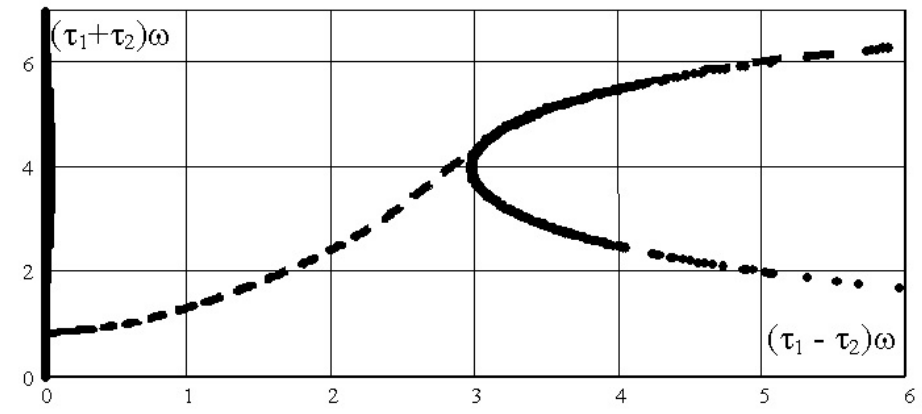

b)

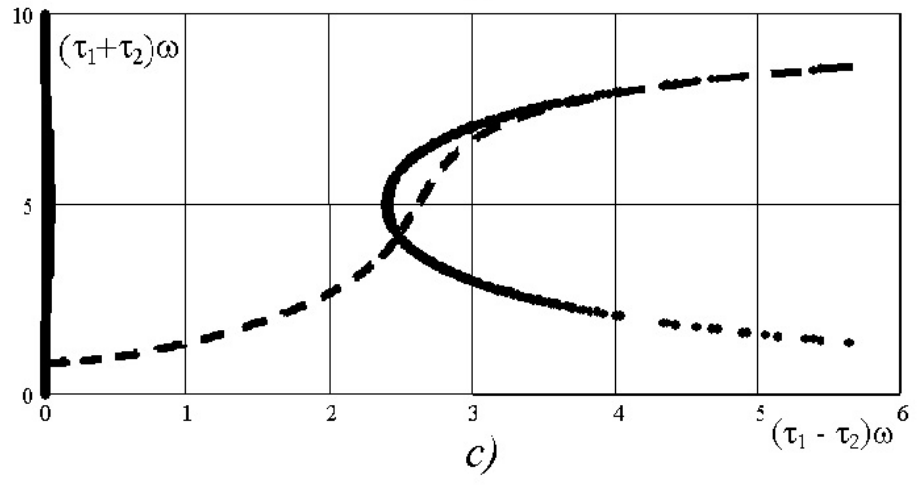

FIG. 6. Results of a numerical solution of a system of transcendental equations (10-11)

5. Theoretical calculation of $2 D$ bifurcation modes in the model $2 D-0$ scillator potential at a finite temperature in an external electric field in the weak dissipation limit for a matrix, possessing metamaterial properties

In case of the quasi-two-dimensional $\mathrm{Au}-\mathrm{QD}$ structures under investigation, we are dealing with a situation where these structures can have the properties of a metamaterial, i.e. the relative permittivity of the heat-bath effectively becomes "negative", and with it the coefficient of interaction of the tunneling particles in the dipoledipole approximation also becomes negative $(\alpha<0)$. As in the numerical analysis of the equations system (10-11), it is possible to identify a bifurcation-tunnel trajectories for certain values of temperature, either the asymmetry parameter of the potential (associated with the magnitude of the external electric field) or the interaction coefficient $\alpha(\alpha<0)$, which depends on the relative permittivity, negative for metamaterials. Numerical analysis of the system (10-11) also makes it possible to reveal a fine structure of the transition in the vicinity of the bifurcation point, i.e. the quantum beat regime for parallel transport of tunneling particles (in addition to the trivial solution (10-11), an additional solution appears).

The result of this numerical analysis is shown in Fig. 7 (and, as is easy to see, differs from the analogous result presented in Fig. 6).

Dependence of the tunneling probability on the electric field strength magnitude is shown in Fig. 8. Realization of the stable 2D bifurcations region has been revealed in Fig. 8b. Fig. 8a shows the beginning of this region. 


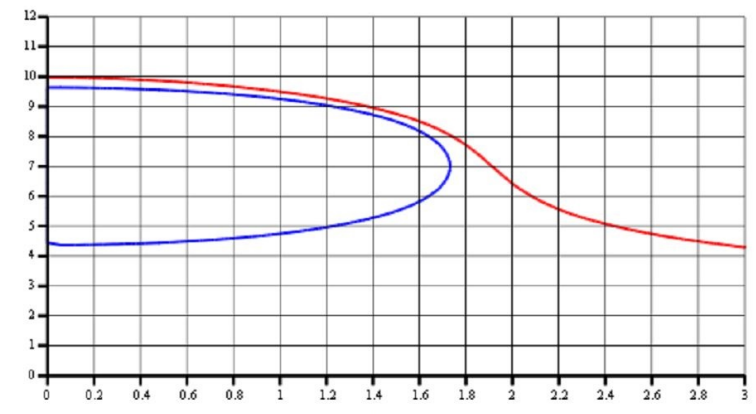

$\mathrm{f} 1, \mathrm{f} 2$

a)

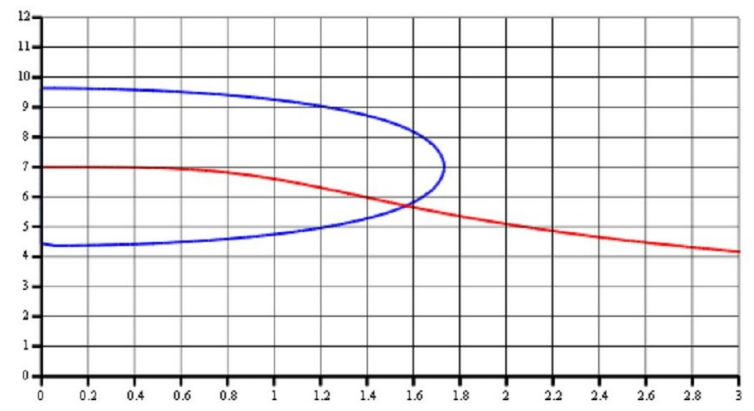

$\mathrm{f} 1, \mathrm{f} 2$

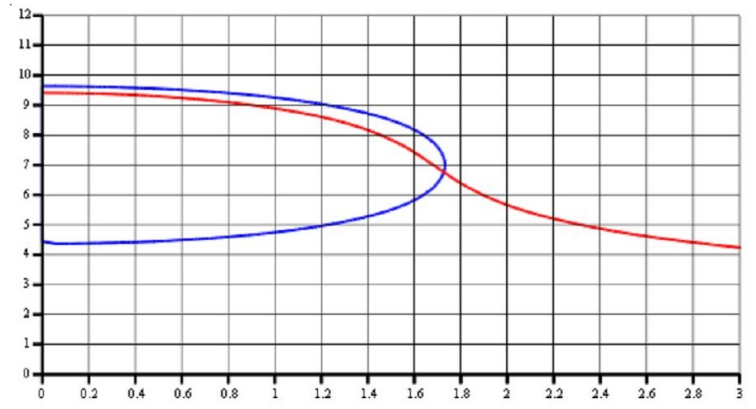

b)

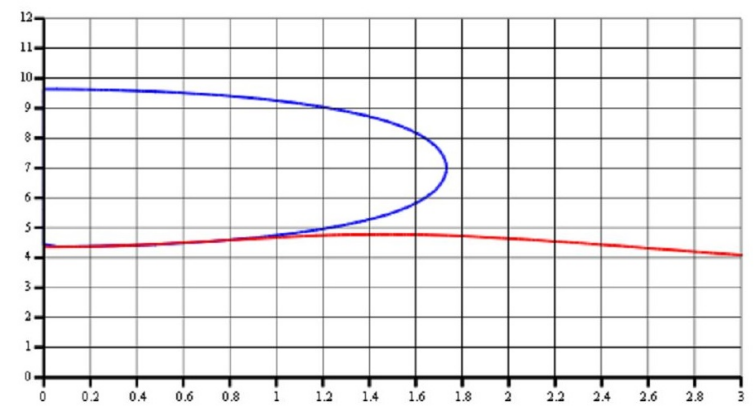

d)

FIG. 7. Numerical solution of the transcendental equations system (10-11) for the case $\alpha<0$. The transition from figure $a$ ) to figure $d$ ) is accompanied by an increase in the strength of the electric field (or the asymmetry parameter of the oscillator potential)
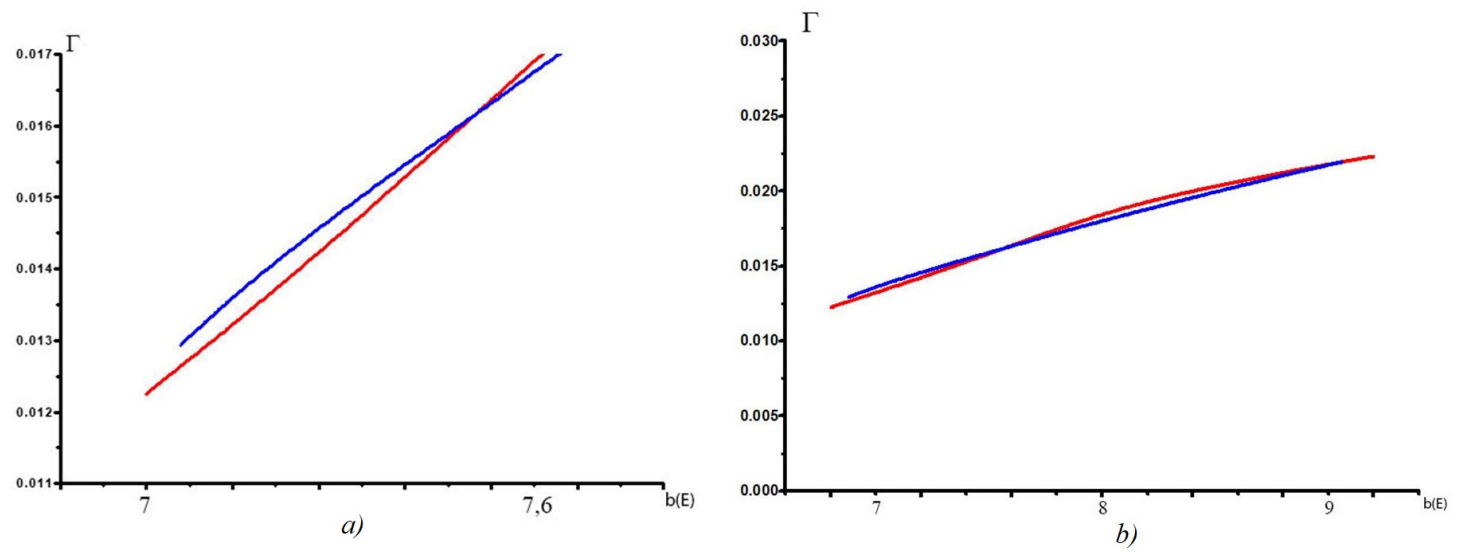

FIG. 8. Dependence of the tunneling probability on the magnitude of the electric field strength

In the vicinity of this point (as in the vicinity of the end point of the stable regime of 2D bifurcations, Fig. 8a and Fig. 8b), the quantum beats mechanism is realized, where the mechanisms of synchronous and asynchronous transfer of tunneling particles compete. Regions for realization of the 2D bifurcations stable effect are identified and the corresponding boundaries for the existence of 2D bifurcations are numerically analyzed when the control parameters (the reciprocal temperature $\beta$, the relative permittivity of the heat-bath $\varepsilon$, and the asymmetry parameter for 2D-potential of the system $b$, which weakly nonlinearly dependent on the strength value of the external electric field) are changed. The corresponding dependence of the field strength range at which stable $2 \mathrm{D}$ bifurcations are realized, depending on the magnitude of the inverse temperature, is shown in Fig. 9.

Fig. 10 presents the "phase diagram" for realization of the stable 2D-bifurcations regime for tunneling current (in case of quasi-two-dimensional $\mathrm{Au}-\mathrm{QD}$ structure) in a matrix of metamaterial depending on the control parameters: the reciprocal temperature, magnitude of the electric field strength (or the asymmetry parameter of 


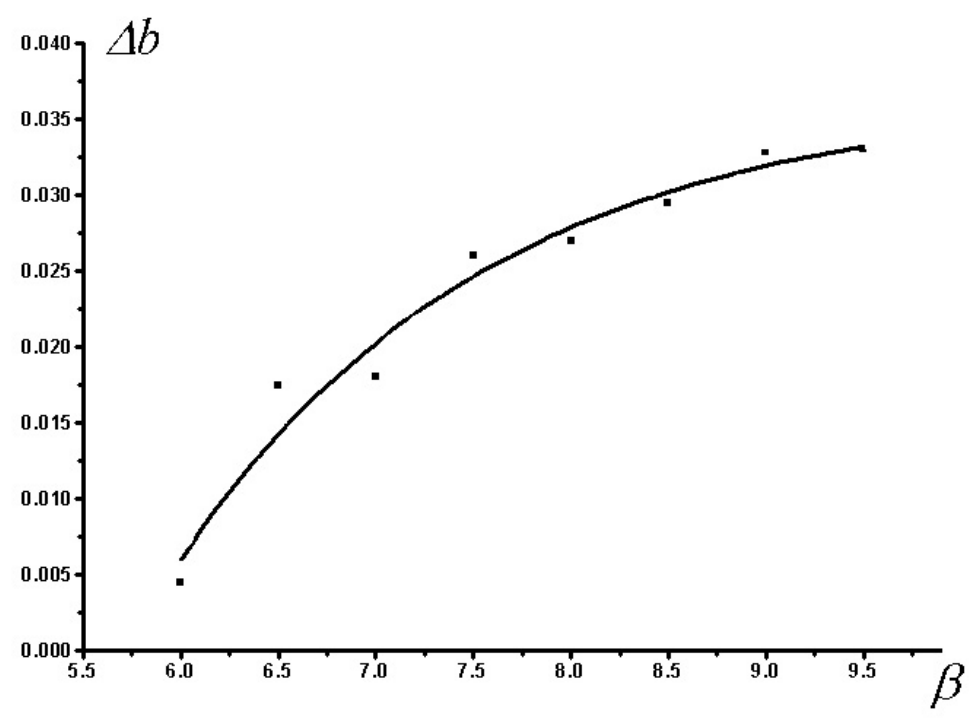

FIG. 9. Dependence of the field strength range at which stable 2D bifurcations are realized, depending on the inverse temperature

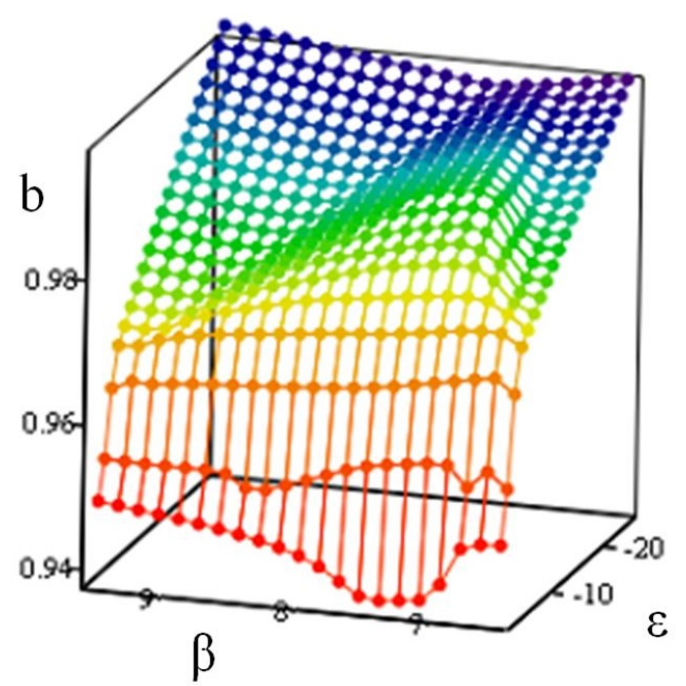

FIG. 10. The phase diagram for realization of the stable 2D-tunneling current bifurcations in a matrix of metamaterial depending on the control parameters: the inverse temperature, the electric field strength (or the potential asymmetry parameter), and the value of the (negative) relative permittivity for the heat-bath matrix

the potential), and the value (negative) of the relative dielectric permeability in the heat-bath matrix, in which the metamaterial is used.

Thus, dependence of the field strength, at which stable 2D bifurcations are realized, on the magnitude of the reciprocal temperature is investigated. In contrast to conventional dielectric matrices, in the case of a metamaterial, the region of stable 2D bifurcations significantly narrows, which is probably due to the inversion of the sign of the tunneling particles interaction.

6. A qualitative comparison of the obtained experimental and theoretical results, as well as a comparative analysis of the 2D-tunneling bifurcations effects both for the case of quasi-one-dimensional and quasi-two-dimensional structures with $\mathrm{Au}-\mathrm{QD}$

As noted in Section 4, solution of the transcendental equations system (10-11) allows us to identify the bifurcation of 2D-tunnel trajectories, at a certain value of the temperature $\beta^{*}$, or the potential asymmetry parameter 
$b^{*}=b / a$ associated with the magnitude of the applied electric field, or the interaction coefficient $\alpha^{*}=2 \alpha / \omega^{2}$, (where $\alpha=e^{2} / \varepsilon \varepsilon_{0} R_{0}^{3}$, in particular, depends on the relative dielectric constant of the heat-bath; the problem of studying $2 \mathrm{D}$ bifurcations with dissipation when changing the parameter $\varepsilon$ may be of separate interest). Numerical analysis of the system (10-11) also makes it possible to reveal a fine structure of the transition in the neighborhood of the bifurcation point, namely, the quantum beat regime for parallel transport of tunneling particles. As a result, the probability of 2D tunneling with exponential accuracy is defined as $\Gamma=\exp (-S)$, where $S$ is given by expression (9), taking into account the solution of system (10-11). Since we are interested in a qualitative comparison with the existing tunnel VAC for the system "cantilever needle - gold nanoclusters (quasi-one-dimensional Au-QD structures" (see Section 2), we are interested in the dependence $\Gamma$ on the asymmetry parameter $b^{*}=b / a$. The result of a comparison of this theoretical curve with the experimental $\mathrm{I}-\mathrm{V}$ characteristic is shown below in Fig. 11. But it must be taken into account that we consider two regions of the electric field change: under positive voltage with the realization of the 2D-bifurcation regime; and at a negative voltage with the attainment of a symmetric potential, which in the case of synchronous tunnel transport along parallel coordinates gives, in the doubled pre-exponential factor, a singularity of the single peak type in this case.

The conditions for the model applicability under consideration are due to the approach of a rarefied gas of pairs "instanton-anti-instanton" and have been discussed in [1-4,8-14]. In the model under consideration, the Coulomb effects can be suppressed if the starting energy of a particle in a QD substantially exceeds the energy of the Coulomb repulsion: $U_{0} \gg \frac{e^{2}}{a_{0}+b_{0}}$.

Thus, generalizing the results of Refs. [1,9], we have obtained a qualitative comparison of the theoretical curves for the dissipative 2D-tunneling probability as a function of the applied electric field, taking into account the bifurcation point (at positive voltage) and the presence of a single peak in the case of a symmetric potential (with negative voltage) with separate experimental $\mathrm{I}-\mathrm{V}$ characteristics for the "platinized cantilever needle - quantum dot (gold nanoclusters)" system, obtained by a group of co-authors from Nizhny Novgorod State University, named by N. I. Lobachevsky. These results are shown in Fig. 11.

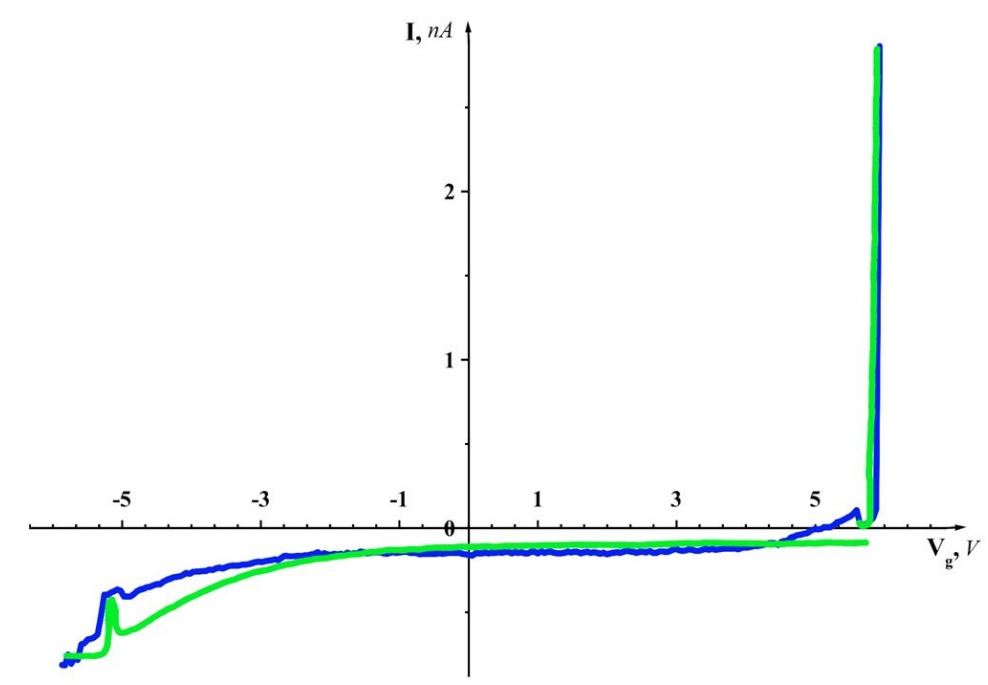

FIG. 11. Comparison of the theoretical curve (green curve) for the 2D-dissipative parallel tunneling probability with the experimental $\mathrm{I}-\mathrm{V}$ characteristic given in Fig. 1b (blue curve)

In addition to the fairly good qualitative agreement between the theoretical and experimental dependences (with the exception of small transition regions), the result of this work allows us to conclude that the stable $2 \mathrm{D}$ bifurcation (changing the tunneling mode from synchronous to asynchronous) predicted in Ref. [1] have been found experimentally. Near this point (a sharp break in the VAC), the small local minimum may be a consequence of the quantum beat regime also described in [1], and which were taken into account in the numerical analysis presented in Fig. 11.

In the case of quasi-two-dimensional $\mathrm{Au}-\mathrm{QD}$ structures possessing metamaterial properties (see Section 3), as noted in Section 5, we obtain on the field dependence of the 2D-dissipative tunneling probabilities not one, but two characteristic breaks. The result of comparing for this field dependence with the experimental $\mathrm{I}-\mathrm{V}$ characteristic (see Fig. 3) is shown in Fig. 12. 


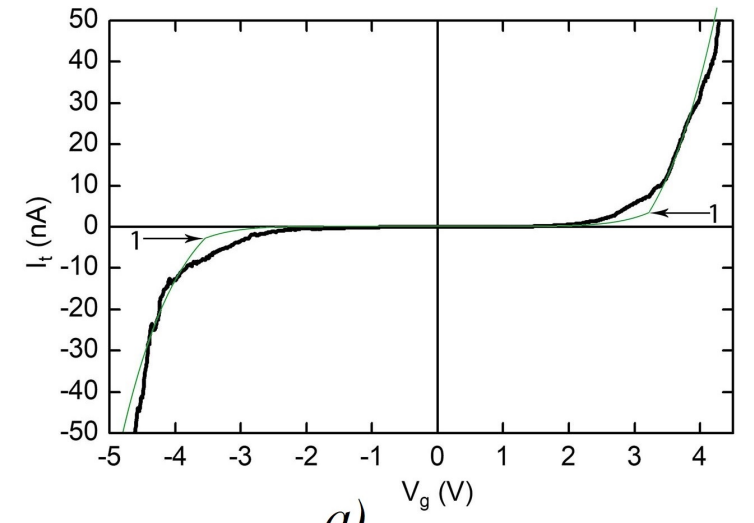

a)

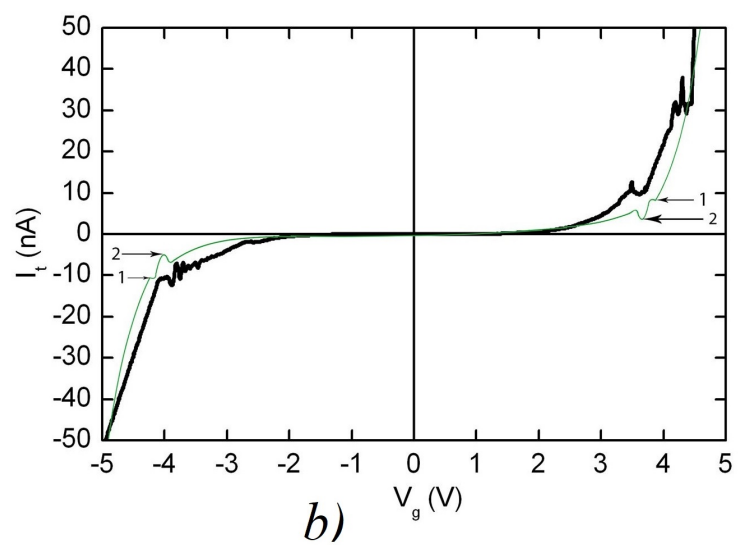

FIG. 12. Comparison of the experimental VAC (black curve) for quasi-two-dimensional Au-QD structures possessing the metamaterial properties (Figs. 2 and 3, with the field dependence of the 2D-dissipative tunneling probability (green curve) calculated in Section 4 in the weak-dissipation limit. Case $a$ ) corresponds to the "smoothed" double kinks or 2D-bifurcations without quantum beats regime. In case $b$ ) in addition to the "smoothed" double kinks (or double 2D-bifurcations regime), the quantum beat regime was also observed in the vicinity of the points of $2 \mathrm{D}$ bifurcations

\section{Conclusion}

The science of the quantum tunneling with dissipation has been developed in the works of A. J. Leggett, I. Affleck, P. Wolynes, A.I. Larkin, Yu. N. Ovchinnikov and other authors $[1-3,8,10-12,14]$. Earlier in his review [10] A. J. Leggett posed the "supertask" of experimental observation for the macroscopic quantum effects of dissipative tunneling. The possibilities and development of the modern nanostructures technology brought the realization of this task closer. We hope that with this work we make our modest contribution to its solution.

\section{Acknowledgements}

Authors thank Prof. A. J. Leggett and Prof. Yu. N. Ovchinnikov (who for the first time together with B. I. Ivlev predicted the 2D-tunneling bifurcations effect) for useful discussions. This work was supported by the Ministry of Education and Science of the Russian Federation (Project No. 3.6321.2017/8.9).

\section{References}

[1] Aringazin A. K., Dahnovsky Yu. I., Krevchik V. D., Semenov M. B., Ovchinnikov A. A., Yamamoto K. Two-dimensional tunnel correlations with dissipation. Phys. Rev. B, 2003, 68, P. 155426(1-12).

[2] Ivlev B. I., Ovchinnikov Yu. N. Decay of metastable states in a situation with close-lying tunneling trajectories. JETP, 1987, 66, P. 378-383.

[3] Benderskii V.A., Vetoshkin E. V., Kats E. I., Trommsdorff H. P. Competing tunneling trajectories in a two-dimensional potential with variable topology as a model for quantum bifurcations. Phys. Rev. E, 2003, 67, P. 026102(1-10).

[4] Zhukovskii V. Ch., Dakhnovskii Yu. I., Gorshkov O. N., Krevchik V. D., Semenov M. B., Smirnov Yu. G., Chuprunov E. V. Rudin V. A., Skibitskaya N. Yu., Krevchik P. V., Filatov D. O., Antonov D. A., Lapshina M. A., Yamomoto K., Shenina M. E. Observed two-dimensional tunnel bifurcations in an external electric field. Moscow University Physics Bulletin, 2009, 64, P. 475-479.

[5] Engheta N. (edt.), Ziolkowski R. W. (edt.) Metamaterials: Physics and Engineering Explorations. John Wiley \& Sons \& IEEE Press, $2006,440 \mathrm{p}$.

[6] Valentine J., Zhang S., Zentgraf T., Ulin-Avila E., Genov D. A., Bartal G., Zhang X. Three-dimensional optical metamaterial with a negative refractive index. Nature, 2008, 455(7211), P. 376-379.

[7] National Science Review. Special issue "Metamaterials Collection”, 2018, 5.

[8] Ovchinnikov Yu. N. Conductivity of granulated metallic films. JETP, 2007, 131, P. 286-290.

[9] Zhukovsky V.Ch., Gorshkov O. N., Krevchik V. D., Semenov M. B., Groznaya E. V., Filatov D. O., Antonov D. A. Controlled dissipative tunneling in an external electric field. Moscow University Physics Bulletin, 2009, 64, P. 27-32.

[10] Caldeira A. O., Leggett A. J. Quantum tunnelling in a dissipative system. Ann. of Phys, 1983, 149, P. 374-456.

[11] Larkin A. I., Ovchinnikov Yu. N. Decay of the supercurrent in tunnel junctions. Phys. Rev. B, 1983, 28, P. $6281-6285$.

[12] Leggett A. J., Ovchinnikov Yu. N., Krevchik V. D., Semenov M. B., Shorokhov A. V. et. al. Transfer Processes in Low-dimensional systems. UT Research Press, Tokyo, 2005, 690 p.

[13] Filatov D., Guseinov D., Antonov I., Kasatkin A. Gorshkov O. Imaging and spectroscopy of Au nanoclusters in yttria-stabilized zirconia films using ballistic electron/hole emission microscopy. RSC Advances, 2014, 4, P. 57337-57342.

[14] Leggett A. J., Ovchinnikov Yu. N., Krevchik V. D., Semenov M. B., Yamamoto K., Filatov D. O. Controllable dissipative tunneling. Tunnel transport in low-dimensional systems. Fizmatlit, Moscow, 2011-2012, 600 p. 